Meta

Journal des traducteurs

Translators' Journal

\title{
In Gutenbergs Fußstapfen: Translatio typographica Zum Verhältnis von Typografie und Translation
}

\section{Jürgen F. Schopp}

Volume 53, numéro 1, mars 2008

Le verbal, le visuel, le traducteur

The Verbal, the Visual, the Translator

URI : https://id.erudit.org/iderudit/017981ar

DOI : https://doi.org/10.7202/017981ar

Aller au sommaire du numéro

Éditeur(s)

Les Presses de l'Université de Montréal

ISSN

0026-0452 (imprimé)

1492-1421 (numérique)

Découvrir la revue

Citer cet article

Schopp, J. F. (2008). In Gutenbergs Fußstapfen: Translatio typographica Zum Verhältnis von Typografie und Translation. Meta, 53(1), 167-183. https://doi.org/10.7202/017981ar

\section{Résumé de l'article}

Depuis Johannes Gutenberg, la dimension visuelle du verbal dans la culture de l'écriture occidentale est marquée par la typographie. L'invention de l'imprimerie typographique a déclenché un développement qui a changé amplement l'apparence des messages verbaux par rapport aux textes écrits à la méthode traditionnelle. Du fait que les textes typographiques servent comme exemple et comme but sur le plan de la publication assistée par ordinateur (PAO), la tradition typographique persiste aussi au temps de l'informatique. Dans le secteur de la réalisation typographique, une division entre typographie d'amateur et réalisation typographique professionnelle en est la conséquence.

La plupart du temps, les textes prévus pour la traduction sont également rédigés en version typographique et font part d'un assemblage de textes soit bicodé soit multicodé. Par ailleurs, les traductions en général en tant que produit intermédiaire sont soumises à une rédaction typographique ou en tant que produit final sont déposées " prêt à publier ». Par conséquent, dans la traduction et dans ses étapes ultérieures, il faut considérer deux aspects de la typographie :

1. Le phénomène sémiotique, c'est-à-dire selon la provenance culturelle en ce qui concerne la typographie, il faut compter avec l'existence des signes spécifiques ainsi que leurs différents significations. En conséquence, cela veut dire aussi que les signes typographiques sont employés de différentes façons selon les cultures. Surtout dans les textes littéraires, l'utilisation de certains signes typographiques peut être l'intention de l'auteur faisant partie de l'oeuvre.

2. L'aspect visuel dans la réalisation de la traduction : les connaissances culturelles des signes typographiques ainsi que les connaissances des conventions typographiques de la culture de départ et de la culture cible (l'orthotypographie) sont aussi indispensables que les connaissances des processus de réalisation typographique des éditions sur le plan professionnel.
Ce document est protégé par la loi sur le droit d'auteur. L’utilisation des services d’Érudit (y compris la reproduction) est assujettie à sa politique d'utilisation que vous pouvez consulter en ligne.

https://apropos.erudit.org/fr/usagers/politique-dutilisation/ 


\title{
In Gutenbergs Fußstapfen: Translatio typographica Zum Verhältnis von Typografie und Translation
}

\author{
J ÜRGEN F. SCHOPP \\ Universität Tampere, Tampere, Finland \\ jurgen.schopp@uta.fi
}

\section{RÉSUMÉ}

Depuis Johannes Gutenberg, la dimension visuelle du verbal dans la culture de l'écriture occidentale est marquée par la typographie. L'invention de l'imprimerie typographique a déclenché un développement qui a changé amplement l'apparence des messages verbaux par rapport aux textes écrits à la méthode traditionnelle. Du fait que les textes typographiques servent comme exemple et comme but sur le plan de la publication assistée par ordinateur (PAO), la tradition typographique persiste aussi au temps de l'informatique. Dans le secteur de la réalisation typographique, une division entre typographie d'amateur et réalisation typographique professionnelle en est la conséquence.

La plupart du temps, les textes prévus pour la traduction sont également rédigés en version typographique et font part d'un assemblage de textes soit bicodé soit multicodé. Par ailleurs, les traductions en général en tant que produit intermédiaire sont soumises à une rédaction typographique ou en tant que produit final sont déposées «prêt à publier ». Par conséquent, dans la traduction et dans ses étapes ultérieures, il faut considérer deux aspects de la typographie:

1. Le phénomène sémiotique, c'est-à-dire selon la provenance culturelle en ce qui concerne la typographie, il faut compter avec l'existence des signes spécifiques ainsi que leurs différents significations. En conséquence, cela veut dire aussi que les signes typographiques sont employés de différentes façons selon les cultures. Surtout dans les textes littéraires, l'utilisation de certains signes typographiques peut être l'intention de l'auteur faisant partie de l'œuvre.

2. L'aspect visuel dans la réalisation de la traduction: les connaissances culturelles des signes typographiques ainsi que les connaissances des conventions typographiques de la culture de départ et de la culture cible (l'orthotypographie) sont aussi indispensables que les connaissances des processus de réalisation typographique des éditions sur le plan professionnel.

\section{ABSTRACT}

Ever since Johannes Gutenberg's times, typography has shaped the visual appearance of the written word in western cultures. Gutenberg's invention gave birth to a development that has changed the appearance of the written message considerably compared to scriptographically manufactured texts. Texts that have been carefully typographically designed still serve as examples both to emulate and also to aim for in desktop publishing. Therefore, the typographical tradition is of essence even in the era of the computer and has resulted in a dichotomy of typographic design (non-professional vs. professional typographic). Hence, all texts that are intended to be translated form part of a bi- or even multi-coded textual network and exist, in one way or another, as typographically designed texts. Equally, a translation project starts as an unfinished product and must undergo, as a rule, some typographical process prior to printing and publication. Therefore, typography is a factor that needs to be taken into consideration under the double aspect of professional translation and the education of translators. 
1. Typography is a semiotic phenomenon, which means that all typographical symbols are specific to their individual cultures and, hence, have different meaning. Also, various cultures will use these symbols with varied frequency. What is more, authors of literary texts may employ typographical symbols intentionally as a special characteristic of the text or as part of it.

2. Typography needs to be considered in the light of the visual appearance of a text, a fact that serves to demonstrate the significance of, firstly, knowledge of a particular culture of typographical symbols; secondly, of typographical conventions of source and target culture (orthotypography); and thirdly, of a solid understanding into the professional production process of typographically produced publications.

\section{MOTS-CLÉS/KEYWORDS}

layout, printing, professional translation, translation process, typography

Translate benötigen einer physischen Gestalt, um entworfen, angefertigt, weitergeleitet, bearbeitet und rezipiert werden zu können - solange sie nur im Gehirn der Translatorin bzw. des Translators existieren, sind sie für Auftraggeber wie Adressat völlig nutzlos. Es ist daher an der Zeit, der visuellen Dimension der Übersetzung nicht nur aus laientypografischer Perspektive Beachtung zukommen zu lassen. Die visuelle Gestalt des Translats kann vorläufig oder endgültig sein. Ersteres liegt vor, wenn - was freilich immer seltener der Fall ist - ein Manu- oder Typoskript angefertigt und anschließend ein „Botschaftsträger“ (Holz-Mänttäri 1984) oder „multimodaler Textverbund“ (Schopp 2005) durch Fachkräfte des grafischen Gewerbes visuell gestaltet wird. Letzteres ist der Fall beim Übersetzen in die layoutformatierte Ausgangstextdatei oder der Anfertigung einer "publikationsfertigen Übersetzung“ (Schopp 2005: 399 u. 405f). Aus translatorischer Perspektive ist dabei die Unterscheidung wichtig, ob die typografische Gestalt des Ausgangstextes konventionell der ausgangskulturellen Orthotypografie (s.u.) folgt und daher beim Translat durch die orthotypografischen Konventionen der Zielkultur zu ersetzen ist, oder ob sie bedeutungskonstituierende visuelle Elemente enthält. Letzteres wird besonders beim literarischen Übersetzen der Fall sein, wenn Autoren bewusst auf die typografische Gestaltung eines Werkes Einfluss nehmen bzw. genommen haben. Es kann z.B. aber auch im Bereich von Touristik-Texten auftreten, indem ausgangskulturelle typografische Zeichen bewusst eingesetzt werden, um dem Translat visuell einen kulturspezifischen „Ton“ oder entsprechendes „Lokalkolorit“ zu verleihen.

Das Verhältnis von Typografie und Translation bzw. Translatologie hat zwei Hauptaspekte. Aus semiotischer Perspektive ist damit zu rechnen, dass typografische Mittel eine Zeichenfunktion ausüben und somit kulturspezifisch geprägt sein können, was unweigerlich zum Auftreten einer Art visueller Kulturspezifik führt. Der zweite Aspekt betrifft die Gestaltung des Translats, das in der heutigen globalen Schreibkultur mit der allgemein verbreiteten Schreibtechnologie Desktop-Publishing so gut wie immer in typografischer Gestalt anzufertigen ist oder diese spätestens im zielkulturellen „Publikat" ${ }^{\text {“1 }}$ erhält. Diese Gestaltung bewegt sich, abhängig von Textsorte und Kommunikationsfeld, auf einer Skala zwischen laientypografischer Textverarbeitung („typografischem Schreiben“) und professionellem typografischem Design. Beim professionellen Übersetzen genügt es daher nicht, dass TranslatorInnen lediglich in der Lage sind, sich laientypografisch zu betätigen, sondern sie müssen über translationsspezifisches typografisches Basiswissen und entsprechende Fertig- 
keiten verfügen, die bereits im Rahmen des Curriculums zu vermitteln sind (vgl. Schopp 2005).

\section{1. "Terra incognita typografica«?}

Wirft man einen Blick auf translatorische Praxis wie Lehre, kann man sich des Eindrucks nicht erwehren, dass für die meisten TranslatorInnen und TranslatologInnen- abgesehen von einigen ,aufgeklärten“ Autodidakten - der Begriff Typografie größtenteils noch terra incognita ist oder dass von einem sehr diffusen bzw. sehr begrenzten Typografiebegriff ausgegangen wird, der das Phänomen entweder auf alle grafischen Texte ausdehnt - auch handschriftliche (Embach 2000: 132: „skriptografisch") - oder sich lediglich auf visuell Auffälliges beschränkt. Das gleiche ist der Fall, wenn die typografische Dimension auf die beim Arbeiten am Computer anfallenden besonderen Arbeitsschritte wie z.B. „Formatierung, Schriftauszeichnung und Schriftart" ${ }^{\text {2 }}$ begrenzt wird.

In der abendländischen Schreibkultur wurde vor Erfindung des Buchdrucks durch Johannes Gutenberg Mitte des 15. Jahrhunderts jede Art von Text von Hand geschrieben - abgesehen vom monumentalen Gebrauch von Schrift und deren durch die Holzschnitttechnik geprägten, relativ groben Wiedergabe in den Blockbüchern des späten Mittelalters. Dabei lassen sich hauptsächlich zwei Qualitätsstufen unterscheiden: die idiografische, individuell ausgeprägte für private und eilige Aufzeichnungen und die kalligrafische, die für repräsentative Texte zum Einsatz kam und den professionellen Schreiber (z.B. im Skriptorium des Klosters) voraussetzte. Beide seien hier unter dem Begriff skriptografisch zusammengefasst. Nachdem Johannes Gutenberg sich bei Satz und Druck seiner 42-zeiligen Bibel noch bemüht hatte, den skriptografischen Charakter des Textes durch ein Inventar von rund 290 unterschiedlichen Buchstabenformen, Buchstabenverbindungen, Abkürzungen etc. beizubehalten, entwickelten seine Nachfolger schon bald eigentliche typografische Druckschriften: im romanischen Sprachraum die Antiqua, in Mitteleuropa die beiden gebrochenen Schriften Fraktur und Schwabacher. Damit hatte sich endgültig eine neue "Schreibtechnologie“ etabliert, die Typografie. Gleichzeitig wurde damit aber auch der Grundstein gelegt für die mit diesen Schriftklassen verbundenen kulturspezifischen Assoziationen (vgl. Schopp 2002a).

Das sprachliche Zeichen Typografie - offensichtlich eine Prägung humanistischer

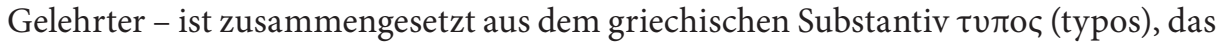
Schlag, Spur, Gestalt, Gepräge, Abdruck bedeutet und im Lateinischen zu typus, im Deutschen zu Typ (vgl. auch Type) wurde, und dem Verb ypaфeıv (graphein), das die Bedeutungen zeichnen, malen, einritzen, graben, schreiben (vgl. dt. gravieren) aufweist. Das Wort bedeutet demnach das „Schreiben“ mit Typen bzw. Lettern, wie es durch Gutenbergs Erfindung in die abendländischen Kultur eingeführt wurde, d.h. den gesamten Gestaltungs- und Herstellungsprozess eines verbalen Textes, wobei unter Gestaltung sowohl die kreative Formgebung als auch der konkrete technische Gestaltungsprozess zu verstehen ist. In dieser Bedeutung findet es sich in Zusammensetzungen wie Buchtypografie, Zeitungstypografie, Werbetypografie etc. oder für die typografischen Teilbereiche Mikro- bzw. Detailtypografie und Makrotypografie.

Im Lexikon der graphischen Technik aus dem Jahr 1970 wird der Begriff Typografie noch definiert als „Die Gestaltung eines Druckerzeugnisses, besonders die von 
Büchern, Broschüren, Zeitschriften, Werbedrucken, Industrie- und Geschäftsdrucksachen, wenn die Anwendung von Schrift überwiegt.“ Die genannten Merkmale reichen unter den heutigen Gegebenheiten nicht mehr zur Begriffsbestimmung aus. Zwar lässt der Hinweis auf die dominierende Rolle der (typografischen) Schrift den Schluss zu, dass zur Visualisierung sprachlicher Zeichen und der diese begleitenden paraverbalen und paralinguistischen Elemente eines Textes (s.u.) selbstverständlich noch weitere Mittel benötigt werden. Doch war die Eingrenzung auf Druckerzeugnisse (Print-Medien) schon damals zu eng, da typografische Schrift außerdem in Film und Fernsehen, auf Laden- und Verkehrsschildern sowie Informationstafeln aller Art vorkommt. Erst recht gilt dies heute nach der Einführung von DesktopPublishing und der starken Verbreitung elektronischer Präsentationsmedien, bei denen das typografische Schriftbild auf digitalem Weg erzeugt wird.

Typografie im eigentlichen Sinne liegt stets vor, wenn ein Text nach dem „typografischen Prinzip“ (Brekle 1997) gestaltet wird. Im weiteren Sinne gehört alles zur Typografie, was die Wirkung dieser Zeichen beeinflusst, also auch die Farbe, die Papierqualität etc.

Das typografische Prinzip besteht darin, dass die Gestaltung eines Textes mit vorgeformten Zeichen (Lettern, Typen) erfolgt, die dann unter Anwendung einer Vervielfältigungstechnik (Druck, digitale Publikationstechniken) bei wiederholtem Auftreten eine identische Gestalt aufweisen. ${ }^{3}$ Die dominierenden Bedeutungsmerkmale beziehen sich hier auf den "Schreibprozess“, nicht auf das entstandene Produkt und seine visuelle Gestalt.

Da aber Typografie nach ihrer linguistischen Entdeckung in den 70er Jahren und ihrer „Demokratisierung“ durch Desktop-Publishing in den 80er Jahren des vergangenen Jahrhunderts in linguistischer und translatologischer Literatur in der Regel als Gestalt eines Textes gesehen wird, nicht als Gestaltungsprozess, darf es nicht verwundern, dass der Typografiebegriff auf jeden veröffentlichten Botschaftsträger ausgeweitet wird, der Textelemente enthält, d.h. auch auf skriptografisch gestaltete Texte mit ihrem deutlich handschriftlichen Charakter. Damit entfernt sich dieser Typografiebegriff allerdings wesentlich von dem des grafischen Gewerbes, aus dem er stammt, da bei diesen Texten (z.B. in Werbetexten und Comics) nicht das typografische Prinzip das optische Erscheinungsbild des Textes prägt, sondern die individuelle, unikale Schriftgestaltung mit folgender z.B. fotomechanischer Vervielfältigung. Allerdings werden gerade in diesen Bereichen in neuerer Zeit pseudohandschriftliche Fonts eingesetzt, was z.T. dazu führt, dass Schriftgrößen gewählt werden, die handschriftlich kaum praktikabel waren, d.h. der skriptografische Charakter solcher Textelemente wird gerade durch die gewählte Schriftgröße ad absurdum geführt ${ }^{4}$ und entpuppt sich letzten Endes als typografisch.

Heute tendiert man dazu, unter Typografie alles zu subsumieren, was mit der technologischen Präsentation von Schrift und der Gestaltung von Texten zusammenhängt, also auch die digitale Herstellung typografischer Schriften und die wissenschaftliche Beschäftigung mit dem Phänomen (vgl. Beinert 2006). Damit ist größtenteils der Status quo wieder hergestellt wie er zu Johannes Gutenbergs Zeit bestand, der sich sowohl seine Arbeitsgeräte (Stempel, Gussform, Lettern) als auch sein Werkzeug (Druckerpresse) schuf, um drucken zu können.

Typografie umfasst als professionelles Handeln grundsätzlich alle Gestaltungsideen, Entscheidungen, Arbeitschritte sowie die Beherrschung und den Einsatz der 
verschiedenen Visualisierungsmittel - in erster Linie Schriftzeichen in den unterschiedlichsten Funktionen und mit unterschiedlichen grafischen Merkmalen - und Gestaltungsprinzipien. Typografie besteht als professionelles Handeln nach der Analyse des Auftrags im zweck- und situationsgerechten Gestalten des Textes bzw. „Publikats“. Dieses erfordert die reflektierte Wahl der geeigneten Grundschrift und der dazu passenden Titelschriften, die (z.T. sprachspezifische) Festlegung von Laufweite, Wortabstand und Zeilenlänge, die damit verbundene Abstimmung von Zeilenabstand und Satzart (Block- oder Flattersatz), die Wahl der Absatzmarkierung und anderer makrotypografischer Parameter - und nicht zu vergessen die sorgfältige Beachtung der kulturspezifischen Orthotypografie.

\section{Visuelle Sprache}

Bei der schriftlichen Translation werden - vielfach unter Beteiligung diverser Fachleute und in einer Zahl von aufeinander abzustimmenden Arbeitsphasen - verbale, d.h. sprachliche Kommunikationsmittel angefertigt, die in den meisten Fällen in typografischer Form präsentiert werden, oft im Verbund mit nonverbalen Botschaftsträgern wie Abbildungen, Skizzen, Grafiken, Lageplänen etc. Diese enthalten ihrerseits nicht selten selbst wieder verbale Elemente. Daher sollte auch für den translatorischen Bereich das Verhältnis von Sprache und Typografie von nicht geringem Interesse sein.

Jeder verbale Text muss entweder über den auditiven oder den optischen Kanal in konkreter Form übermittelt werden, d.h. als gesprochener oder schriftlicher Text. Damit haben wir zwei Manifestationsformen der Sprache, eine vokale und eine grafische (vgl. Nöth 2000: 295) bzw. eine auditiv-verbale und eine visuell-verbale letztere in den Versionen "Hand-made" und "Machine-made" (vgl. Twyman 1982: 7), wofür ich mit Bezug auf das optische Erscheinungsbild „skriptografisch“ und „typografisch“ sage. Da in unserer Schriftkultur der Großteil aller Texte in typografischer Gestalt angefertigt und rezipiert wird - für viele ist die Times New Roman bereits so etwas wie eine persönliche Handschrift -, tendiere ich dazu, den typografisch gestalteten Text als Prototyp für visuell-verbale Sprachprodukte anzusehen.

Aus translatorischer Perspektive ist es außerdem wichtig zu berücksichtigen, dass typografisch gestaltete Texte sich hinsichtlich ihrer visuellen Qualität seit der Etablierung von Desktop-Publishing als allgemeiner Schreibtechnologie zwischen den beiden Polen „laientypografische“ und „professionelle typografische Gestaltung“ bewegen. Bei großem Repräsentationswert des Publikats wird in der Regel neben hohen Anforderungen an dessen materieller Ausstattung ein visuelles Qualitätsniveau erwartet, das sich mit laientypografischen Kenntnissen und Fertigkeiten nicht erreichen lässt.

Für Saussure war die Schrift noch ausschließlich ein Zeichensystem zur optischen Darstellung eines anderen Zeichensystems, der gesprochenen Sprache (vgl. 1931/1967: 28), also ein Zeichensystem 2. Ordnung. Heute setzt sich jedoch immer mehr die Auffassung durch, dass Schriftsprache und Lautsprache zwei, zwar in großen Teilen aufeinander beziehbare, in ihren Einsatzbereichen jedoch eigenständige Subsysteme bzw. Kommunikationsmittel mit unterschiedlichen, aber komplementären kulturellen Funktionen sind (vgl. Nöth 2000: 361), wobei selbstverständlich bei einer Reihe von Textsorten der Fall eintritt, dass gesprochene Sprachelemente visualisiert werden müssen (z.B. in Romanen, Erzählungen, Comics, Interviews). 
Schrift in typografischer Gestalt ist in einer Vielzahl von Arten und Varianten (Schnitten) zwar das typografische Vertextungsmittel par excellence zur Visualisierung verbaler Elemente, doch kommen zur Gestaltung eines komplexen Textes eine

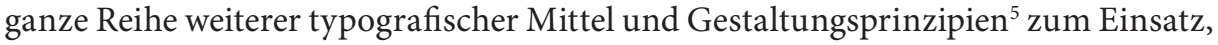
die im Verbund ein Textgebilde entstehen lassen wie wir es heute als selbstverständlich voraussetzen. Solch ein typografischer Text ist ein komplexes Gewebe unterschiedlichster Zeichen, darunter nicht wenigen, die von Laien oft nicht bewusst wahrgenommen werden.

In mündlicher Kommunikation werden die verbalen Elemente -, d.h. Elemente, die Lexik, Idiomatik und Grammatik eines Sprachsystems repräsentieren -, begleitet von prosodischen Phänomenen wie Intonation, Betonung und Pausen, die grammatisch bedingt sind und daher zum linguistischen System einer Sprache gehören (Tiittula 1992: 43); solche Phänomene können als paraverbal bezeichnet werden. Hinzu treten weitere prosodische Phänomene des Stimmgebrauchs wie z.B. der Wechsel in der Stimmhöhe, die Lautstärke, das Tempo und die Stimmlage sowie nicht grammatisch bedingte Pausen. Diese verleihen der Botschaft eine affektive Komponente und werden als paralinguistisch bezeichnet, da sie nicht zum Sprachsystem gehören (ibid.), jedoch unabdingbarer Bestandteil jeder gesprochenen Äußerung sind. Alle werden über den auditiv-vokalen Kanal vermittelt. Begleitet wird mündliche Kommunikation stets auch von nonlinguistischen Phänomenen wie Kinesik (Gestik und Mimik) und Proxemik (Redeabstand und -raum), wahrgenommen über den visuellen Kommunikationskanal.

Alle diese Phänomene und Beziehungen müssen bei der schriftlichen Kommunikation über den visuell-grafischen Kanal vermittelt werden. Dabei ist es selbstverständlich, dass je nach Textsorte und Genre typografische, bildhaft-nonverbale oder verbale Mittel zum Einsatz kommen. Wenn z.B. im Comic (hierzu ausführlich Kaindl 2004: 220ff) das plötzliche Ansteigen der Lautstärke durch den Wechsel der Schriftgröße gleichsam ,ikonisiert“ wird wie im handgeletterten französischen Original ${ }^{6}$ in Abb. 1 (in der typografischen deutschen Übersetzung sowie den ebenfalls handgeletterten englischen und finnischen Übersetzungen durchweg nicht beachtet), wird dies in belletristischer Literatur konventionell entweder durch entsprechende verbale Mittel ausgedrückt oder behutsam typografisch angedeutet, z.B. durch Versalien der Grundschrift. Prinzipiell bietet der typografische Präsentationsmodus aber wesentlich mehr sowie differenziertere und subtilere Ausdrucksmöglichkeiten als der skriptografische.
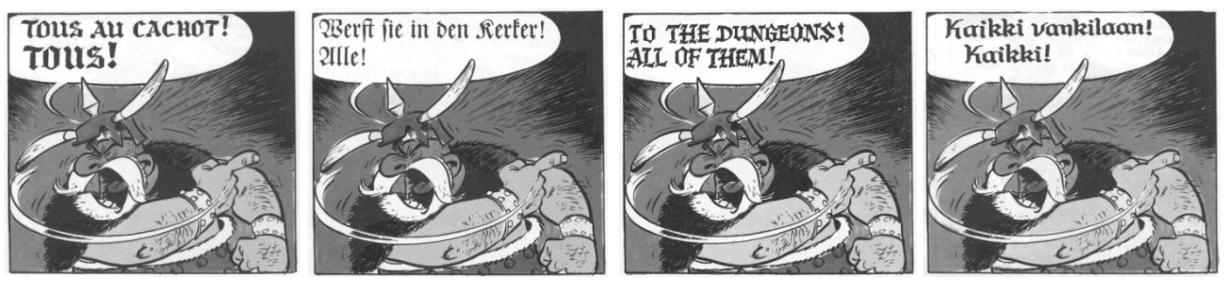

Abb. 1: Asterix und die Goten - Verlust paralinguistischer Information in den Übersetzungen 
Ein Problem ergibt sich aus dem Bedeutungsumfang von nonverbal, das einerseits bestimmte Begleitphänome verbaler Kommunikation umfasst, andererseits aber als Oberbegriff für alle nonverbalen Kommunikationsmittel wie Abbildungen, Pläne und Landkarten dient. Diese können prinzipiell als autonome Kommunikationsformen auftreten, kommen aber häufig als Hilfsmedium zusammen mit schriftlichen Kommunikationselementen vor oder bilden wie z.B. in Comics und Karikaturen das Hauptmedium. Es ist also strikt zu trennen zwischen nonverbalen Elementen, die im „semiotischen Kontext“ (Nöth 2000: 298) zum Kommunikationssystem Sprache stehen, die sprachliche Kommunikation begleiten oder ergänzen, und nonverbalen Kommunikationseinheiten, die im Rahmen nicht-sprachlicher Kommunikation eingesetzt werden bzw. sprachliche Kommunikationsinhalte ersetzen.

Warum kommt es nun aber immer wieder zu dem Phänomen, dass die zweifache - verbale ${ }^{7}$ und typografische - Kodierung des sprachlichen Zeichens (Jegensdorf 1980: 26) nicht bewusst wahrgenommen wird, sondern letztere zum verbalen Zeichen selbst wird, dessen zusätzliche visuelle Information u.U. unbeachtet bleibt? Schon Saussure hatte erkannt, wie stark die Dominanz des schriftlich fixierten Wortes den Sprachbegriff prägt und wie schwer die bewusste Unterscheidung von verbalem Inhalt und visueller Gestalt fällt:

Auch heute noch verwechseln gebildete Leute die Sprache mit ihrer Orthographie [...] Man vergißt zuletzt, daß man sprechen lernt, ehe man schreiben lernt, und das natürliche Verhältnis ist umgedreht. (1931/1967: 30)

Daran hat sich bis heute im Grunde wenig geändert. Die typografische Gestalt sprachlicher Produkte ist für uns moderne Menschen meist schon von früher Kindheit an so selbstverständlich, dass wir sie nicht selten mit Sprache gleichsetzen. ${ }^{8}$ Diese Gleichsetzung des sprachlichen Zeichens mit seiner grafischen Gestalt ist insoweit verständlich, als in der Rezeptionsphase ja beide als eine Einheit wahrgenommen werden bzw. die verbalen Zeichen nur über ihre (typo)grafische Realisierung rezipiert werden können. In Zusammenhang damit ist die Bildung von Wortbildern zu sehen, die - im Gedächtnis gespeichert - den Leseprozess beschleunigen und für ein rationelles Lesen unabdingbar sind, da sie eine Art „ideografisches Lesen“ ermöglichen (vgl. Schopp 2005: 228f).

Hierin mag ein Grund liegen, warum es bei der Translation immer wieder zur Vernachlässigung der grafischen bzw. typografischen Gestalt des Textes kommt -, sei es, dass paraverbale und paralinguistische Elemente nicht erfasst und im Translat unterschlagen werden, wie Kaindl (2004: 220ff) überzeugend für die Textsorte Comic nachweist ${ }^{9}$-, sei es, dass in der Übersetzung die Ganzheitlichkeit eines literarischen Kunstwerkes zerstört wird, wie Oittinen anhand der Übersetzung von Werken der finnlandschwedischen Autorin und Künstlerin Tove Jansson aufzeigt (1993: 114) -, oder dass vom Autor bewusst in eines seiner Werk integrierte typografische Ausdrucksmittel wegfallen, wie ich es anhand Mark Twains A Connecticut Yankee in King Arthur's Court demonstriere (Schopp 2005: 376-384; vgl. unten Abb. 4).

Auch wenn in der Rezeptionsphase das sprachliche Zeichen als Einheit aus verbalem Inhalt und visueller Gestalt wahrgenommen wird, ist es methodisch sinnvoll, zu Analysezwecken und für die Produktionsphase beide Ebenen zu trennen; dies umso mehr, als im Regelfall unterschiedliche Fachkompetenzen und Berufe an der Herstellung beteiligt sind und zum Gesamt des Botschaftsträgers beisteuern. 


\section{Tektonik, Textur, Typografie - das »TT+T-Modell»}

Jeder, besonders aber der typografisch gestaltete Text ist ein Gewebe von Zeichen unterschiedlicher Ebenen, die einander bedingen, aufeinander Bezug nehmen, sich gegenseitig ergänzen und so zusammen die Botschaft an den Adressaten vermitteln. Dies veranschaulicht das von mir so genannte „TT+T-Modell“ (Schopp 2005: 59-61) in Abb. 2, das die von Holz-Mänttäri auf Designtexte und Translate angewendete Dichotomie von Tektonik und Textur um die visuelle Dimension Typografie erweitert.

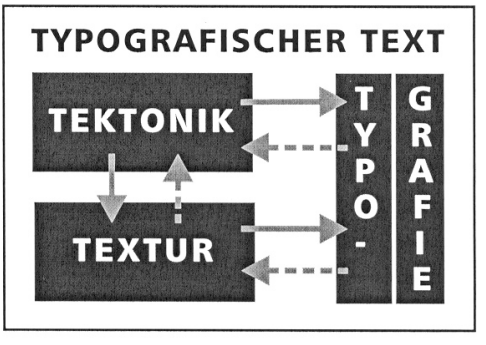

Abb. 2: das »TT+T-Modell«

Unter Tektonik versteht Holz-Mänttäri die „Inhaltsstruktur“ (1984: 128) bzw. „das tragende Gerüst eines Designtexts“ (1993: 311), bestehend in Auswahl und Anordnung der kommunikativen Teilhandlungen. Ich rechne unter funktional-kommunikativem Aspekt außerdem die textsortentypische Koordination hierarchisch geordneter Teiltexte und Textteile sowie das Auftreten para- und metatextueller Elemente wie z.B. dem „lebenden Kolumnentitel“ in wissenschaftlichen Publikationen hinzu.

Den Begriff Textur bezieht Holz-Mänttäri (1984: 77) zunächst in Anlehnung an Jegensdorf (1980) auch auf die visuelle Gestalt, definiert den Terminus aber im Folgenden ausschließlich für die sprachliche Form: „Textur nennen wir die Strukturierung der Ausdrucks- und Verknüpfungsmittel eines Textes, die ihm im Bereich der Formen Kohärenz verleiht“ (1984: 134). Die Erklärung könnte - wie oben bereits angedeutet - darin bestehen, dass auch hier der visualisierte sprachliche Zeichenkörper als das sprachliche Zeichen per se aufgefasst wird. Damit wird aber die visuelle Gestalt zum einen nicht als eigenständiges Phänomen in ihrer semiotischen Zeichenhaftigkeit gesehen und zum andern nicht als Resultat einer gesonderten professionellen Handlung erkannt.

Ich verstehe unter Textur die Gesamtheit aller verbalen Mittel, die aufgrund der Tektonik ausgewählt und angeordnet sind (lexikalische, phraseologische, syntaktische und stilistische Einheiten, Verweis- und Verknüpfungselemente, aus denen sich die Mikro- und Makrostruktur des Textes aufbaut) und aus der sich der verbale Inhalt des Textes ergibt.

Unter dem Blickwinkel der professionellen Gestaltung eines Translats bzw. eines zielkulturellen Publikats ist daher die Erweiterung des Holz-Mänttärischen Modells um die Dimension Typografie sinnvoll und notwendig.

Diese dritte Ebene des Botschaftsträgers bildet die optische Gestalt des Textes als Resultat der Visualisierung von verbalen, paraverbalen und paralinguistischen Elementen. Durch typografische Mittel wird sowohl die Textur als auch die Tektonik des Textes sichtbar gemacht (in Abb. 2 durch die Pfeile symbolisiert). In einigen 
Fällen können typografische Zeichen bedeutungskonstituierend auftreten, indem sie durch Aktivierung von kulturspezifischem Wissen kontextuell in den Gesamttext Bedeutungselemente integrieren, die so nicht verbalisiert werden müssen (Abb. 3). Wenn in der Anzeige anlässlich der österreichischen Parlamentswahlen von 1994 beim Textelement „Andere tun es auch.“ ein Wechsel der Schriftart zur Frakturschrift vorgenommen wird, so ist die Botschaft mit Bezug auf die kulturspezifisch bekannte Relation von gebrochenen Schriften und Nazi-Ideologie jedem Österreicher und Deutschen klar: „Die Rechtsextremen gehen jedenfalls wählen.“ Diese Bedeutungsverschiebung wird allerdings vielerorts in der Welt nicht nachvollzogen, wo gebrochene Schriften weiterhin das Merkmal „typisch deutsch“ tragen (vgl. Schopp 2002a). Stellvertretend stehe hier die Titelseite des Stadtführers von Kairo aus den 90er Jahren. Während in der englischen Ausgabe der Stadtname Kairo in einer modern bis exotisch wirkenden Schrift gesetzt wurde, präsentiert er sich in der deutschsprachigen Ausgabe in Gotisch (Abb. 4).

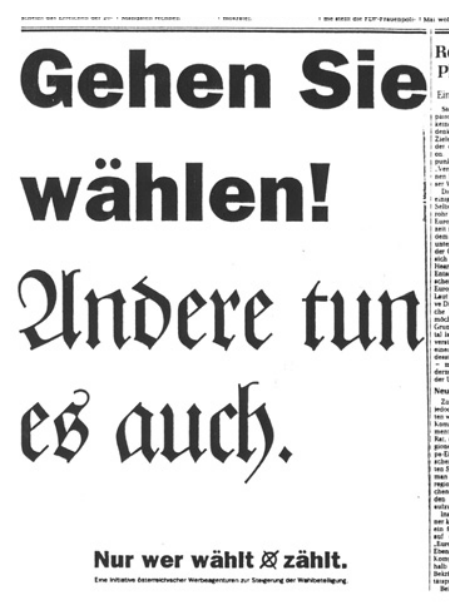

Abb. 3: Politisch motivierter Wechsel des Schriftcharakters, durch den dem verbalen Inhalt visuell eine semantische Bedeutung hinzugefügt wird
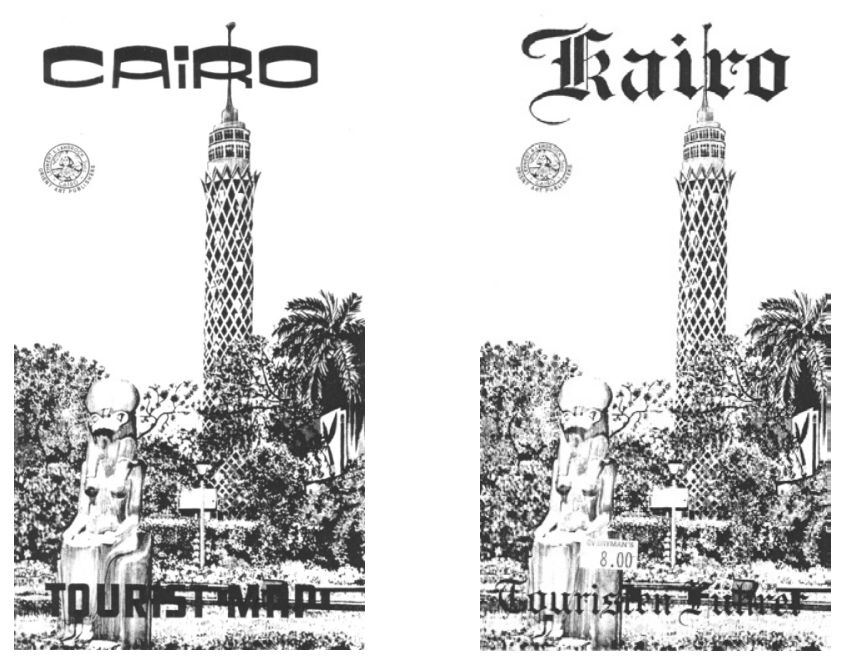

Abb. 4: Kairo auf Deutsch - typografisches Stereotyp oder Kulturspezifik? 


\section{Typografische Zeichenfunktionen}

Entsprechend der Komplexität von Texten als Botschaftsträgern weist Typografie eine Vielzahl von Funktionen auf. Neben den von Bühlers Organonmodell ableitbaren kommunikativen Grundfunktionen der Darstellung, des Ausdrucks und des Appells sowie der von Willberg/Forssman auf unterschiedliche Leseanlässe bezogenen acht Funktionen der Lesetypografie (hierzu Schopp 2005: 168-172) finden sich eine ganze Reihe weiterer Aspekte und Funktionen. Hier sei vor allem auf den Bezug typografischer Einheiten zu verbalen, paraverbalen, paralinguistischen und nonverbalen Elementen und ihren Beitrag zur Konstitution von „Bedeutung“ eingegangen, d.h. auf ihre semiotische Funktion.

Prinzipiell ist davon auszugehen, dass durch typografische Mittel alle sprachlichen Ebenen sowie die begleitenden paraverbalen und paralinguistischen Phänomene abgebildet werden können, wobei es von der Textsorte oder vom Genre abhängt, ob etwas typografisch visualisiert oder (metasprachlich) verbalisiert werden muss. In belletristischen Werken z.B. werden Affektivität und Emotionalität von Äußerungen in der Regel durch verbale Mittel gekennzeichnet, während in einem Comic das grafische Erscheinungsbild der Äußerung diese gleichsam „ikonisiert“ und in das betreffende Bild integriert (hierzu Kaindl 2004: 228).

Jegensdorfnenntim Hinblickaufden Beitrag des Visuellen zur Bedeutungs(ab)bildung des Visualisierten fünf Grundfunktionen des Visuellen: (1) bedeutungs-gliedernd, (2) bedeutungs-akzentuierend, (3) bedeutungs-ergänzend, (4) bedeutungs-aufbauend und (5) bedeutungs-,,frei“ (1980: 71-86). Hier gehe ich von nur drei Hauptfunktionen aus, da ich die auf der Grundlage eines sekundären Zeichensystems erfolgende Darstellung von Tektonik und Textur durch Typografie als Basisfunktion und konstituierend für visuell-verbalen Sprachgebrauch ansehe. Die Frage ist demnach, ob und inwieweit durch die gewählten typografischen Elemente zum semantischen Inhalt Bedeutungselemente hinzutreten, d.h. ob neue, weitere Inhalte dem visualisierten sprachlichen Zeichen hinzugefügt werden. In Bezug auf das Zeichenniveau wäre die primäre Ebene, bei der das Zeichen bzw. der Zeichenkörper direkt zur Konstitution von Bedeutung beiträgt, zu unterscheiden von der sekundären Ebene, bei der das Zeichen auf ein Zeichen der ersten Ebene verweist; schließlich noch die tertiäre Verwendung, bei der die Gestalt des visuellen Zeichens nicht auf dessen verbalen Inhalt bezogen wird, sondern gewissermaßen ein Eigenleben führt und für sich gesehen z.B. als dekorative Form eingesetzt wird (Jegendorfs „,bedeutungs-",freie“ Funktion).

Im Hinblick auf den Beitrag typografischer Elemente zur Konstruktion von Bedeutung genügt es, folgende Fälle zu unterscheiden: 1. die (relativ) bedeutungsneutrale typografische Visualisierung (visuell-verbale Basisfunktion); 2. die Hinzufügung von Bedeutung durch typografische Elemente zum verbalen Inhalt (das typografische Zeichen wird somit zum primären Zeichen) sowie 3. als einen Sonderfall die ,ikonische“ Abbildung verbaler Inhalte durch typografische Elemente. Als 4. und weiterer Sonderfall ist die Abbildung von „Textbildern“ zu sehen, z.B. wenn die Gestalt älterer typografischer Texte "gezeigt" werden soll. Im Folgenden seien die einzelnen Punkte näher ausgeführt:

1. Als typografische Basisfunktion hat prinzipiell die Visualisierung des Textes zu gelten - von Laien oft nicht wahrgenommen, da für selbstverständlich bzw. für 
das schriftsprachliche Zeichen per se gehalten (s.o.). Durch den direkten Bezug auf ein anderes Zeichen liegt eine „sekundäre“ Zeichenfunktion vor. Die typografische Gestalt ist (relativ) bedeutungsneutral und beschränkt sich in erster Linie auf die Visualisierung verbaler Zeichen. ${ }^{10}$ Neben der Darstellung verbaler Zeichen finden sich durch Anwendung bestimmter Hervorhebungstechniken - in Belletristik meist kursiver Schriftschnitt - auch paraverbale und paralinguistische Elemente.

Ein wichtiges Moment innerhalb dieser Funktion ist die Gestaltung des Textes unter dem Postulat der Lesbarkeit, die solides typografisches Fachwissen und Fachkönnen voraussetzt (über das lediglich auf laientypografischer Basis arbeitende TranslatorInnen eben nicht verfügen), da es hier darum geht, die typografischen Parameter Schriftcharakter, Schriftgröße, Laufweite, Wortabstand, Zeilenlänge und Zeilenabstand sprachspezifisch sorgfältig aufeinander abzustimmen.

Ein dritter Bereich - ebenfalls aus translatorischer Perspektive zu berücksichtigen - ist die schriftkulturspezifische Orthotypografie, die sich in der Gestalt, der Frequenz und im Einsatz typografischer Einzelzeichen äußert, durchaus aber auch im kulturspezifischen Gestaltungsniveau eines Publikats bestehen kann, d.h. die unter Umständen textsortenspezifisch unterschiedliche qualitative Realisierung des Layouts in den beteiligten Translationskulturen (s.u.).

2. Durch ein typografisches Mittel wird dem sprachlichen Zeichen ein ergänzendes oder modifizierendes Moment beigefügt, das zur Konstruktion einer neuen Bedeutung führt; in diesem Fall wird das typografische Zeichen zum „primären Zeichen“. Dies ist der Fall, wenn wie in Abb. 3 (s.o.) ein kulturspezifisch interpretierter Schriftcharakter in Verbindung mit spezifischen Inhalten auftritt. Weniger spektakuläre Fälle sind die typografische Markierung von Bedeutungsklassen in einem laufenden Text wie z.B. Namen, Buchtitel, Begriffe, Fremdwörter durch kursiven Schriftschnitt (Walker 2001: 12 "distinction" und "differentiation").

3. Durch die Typografie wird das verbal Geäußerte ein zweites Mal kodiert ${ }^{11}$ - es handelt sich aus semiotischer Sicht um „fallende Semiosen“ (Wehde 2000: 179), da im Gegensatz zu „steigenden Semiosen“ wie in Abb. 3 keine neuen Bedeutungselemente auftreten (Beispiele in Schopp 2005: 173, Abb. 37), sondern Inhaltsmerkmale gleichsam „ikonisiert“ werden, um z.B. einen größeren Aufmerksamkeitseffekt zu erzielen.

4. Und schließlich noch ein vierter Fall, der darin besteht, dass andere (z.B. ältere) Zustände eines Textes durch typografische Mittel als „Text-Bild“ abgebildet werden sollen - ebenfalls eine ikonische Funktion, die sich allerdings nicht auf den verbalen Inhalt bezieht, sondern dessen visueller Gestalt. Als Beispiel mag hier eine Abbildung aus Mark Twains A Connecticut Yankee in King Arthur's Court dienen, die das typografische Niveau einer fiktiven „ersten Zeitung“ abbilden soll (Abb. 5). Dieses Niveau entspricht offensichtlich dem jener kleinen Lokalblätter des Mittleren Westens der USA, das Samuel Langhorne aus eigener Erfahrung kannte - immerhin hatte er als Zwölfjähriger in der Druckerei seines Bruders Orion in seiner Heimatstadt Hannibal am Mississippi eine Schriftsetzerlehre begonnen und nach derem Abschluss u.a. eine Zeit lang im Mittleren Westen als Redakteur gearbeitet. 


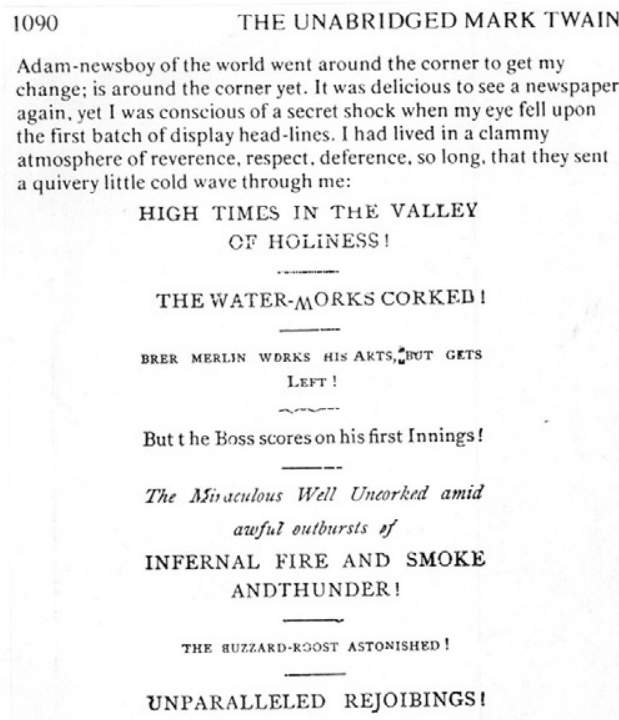

- and so on, and so on. Yes, it was too loud. Once I could have enjoyed it and seen nothing out of the way about it, but now its note was discordant. It was good Arkansas journalism, but this was not Arkansas. Moreover, the next to the last line was calculated to give offence to the hermits, and perhaps lose us their advertising. Indeed. there was too lightsome a tone of flippancy all through the paper.

It was plain I had undergone a considerable change without noticing it. I found myself unpleasantly affected by pert little irreverencies which would have seemed but proper and airy graces of speech at an earlier period of my life. There was án abundance of the following breed of items, and they discomforted me:

$$
\text { Local Smoke ani Cinders. }
$$

Sir Jauncejor met up with old King Vgrivance of Ireland unexpectedly last weok over on the moor south of $\mathrm{Si}_{1}$ Balmoral le Merveilleuse's hog dasture. The widow has been notified.

\section{A CONNECTICUT YANKEE}

Expedition No. 3 will start adout the first of next mgnth a search f $8 \mathrm{r}$ Sir Sagramour le Desirous. It is in comand of the renowned Knight of the Ked Lawns, assissted by Sir Persant of Inde, who is competegt. intelligent, courteous, and in every mav a brick, and further assisted by Sir Palamides the Saracen, who is no huckleberry hiuself. This is no pic-nic, these boys mean busine\&s.

The readers of the Hosannah will regrec to learn that the hadndsone and popular Sir Charolais of Gaul, who during his four weeks' stay at the Bull and Halibut, this city, has won every heart by his polished massers and elegant c Tnversation, will pull out to-day for home. Give us another call, Charley! The bdsiness end of the funeral of the late Sir Dalliance the duke's son of Cornwall, killed in an encounter wich the Giant of the Knotted Bludgeon last Luesday on the borders of the Plain of Enchantaiciat was in the hands of the ever affable and eycient Mumble, prince of unzertakers, than whom titere exists none by whom it were a more satisfying pieastire to have the last sad ofitices performed. Give him a trial.

'The c'rivial thanks of the Hosannah office are due, from editor down to devil, to the ever courteous and thoughtful Lord High Stew 1 of the Palace's Thrid Assistant V f for several sauc $\nmid$ s of ice cream a quality calculated to make the ey of the recipients humil with gr ade; and it done it. When this administration wants to chalk up a desirable name for early premotion, the Hosannah would like a chance to sidgest.

\section{Abb. 5: Text als Bild - Visualisierung von Gestaltetem}

Zum Abschluss dieses Abschnittes sei ein Beispiel für die Polysemie typografischer Mittel aufgeführt: Der kursive Schriftschnitt (Italic) wird konventionell im Rahmen des „integrierten Auszeichnens“ häufig als Hervorhebungsmittel parallel zum Normalschnitt einer Schrift verwendet, um prosodische Elemente (z.B. Betonung) zu markieren (vgl. Walker 2001: 12: "emphasis"). In wissenschaftlichen Abhandlungen kann er aber auch dazu dienen, im laufenden Text Begriffe, Namen und Titel zu kennzeichnen. Bei einer Reihe von Publikaten werden ganze Teiltexte in Kursiv gesetzt (z.B. das Vorwort). Schließlich ist denkbar, dass Kursiv bei passender Thematik und nicht allzu umfangreicher Textmenge in einer Broschüre als Grundschrift dient oder in einem mehrsprachigen Flyer eine der Sprachversionen markiert. Daneben finden sich - vor allem in experimenteller Literatur - unikale, nicht konventionalisierte Einsatzmöglichkeiten, z.B. wenn in einem Roman wie Uwe Johnsons Mutmaßungen über Jakob von 1959 „die Kursive ich-perspektivisches, die Grundschrift jedoch auktoriales Erzählen bezeichnet“" (Jegensdorf 1980: 80). 


\section{Visuelle Kulturspezifik}

Bei der Herstellung eines Translats ist vor allem aus zwei Gründen auf die Typografie des AT bzw. des ZT zu achten. Der erste Grund besteht darin, dass bei einer Reihe von Textsorten neben verbalen Zeichen auch paraverbale und paralinguistische Elemente grafisch bzw. typografisch visualisiert werden. Dies erfordert eine Sensibilität der Translatorin, da - wie Abb. 1 beweist - relevante visuelle Information leicht „übersehen“ wird.

In diesem Abschnitt sei näher auf einen weiteren Grund eingegangen: Da typografische Mittel Zeichencharakter besitzen - auch wenn Typografie an sich eher als „Zeichensystemoid“ bezeichnet werden muss ${ }^{12}$ - ist damit zu rechnen, dass sie kulturspezifisch eingesetzt werden bzw. dass kulturspezifische typografische Mittel existieren. Damit aber besteht die Möglichkeit, dass sie prinzipiell als Translationsproblem auftreten können, sei es aufgrund ihrer kulturspezifischen Ausprägung, ihrer Gebrauchsfrequenz oder aufgrund einer kultur- bzw. autorenspezifischen Verwendung.

Solche Fälle, an denen sich die Relevanz typografischer Kenntnisse beim Translationsprozess zeigt, müssen in der AT-Analysephase erkannt werden. Daher ist bei der visuellen Analyse des AT sowie bei der Gestaltung des ZT besonders auf die Kultur- und Sprachspezifik der Schriftsysteme, der einzelnen typografischen Zeichen, der kulturspezifischen Anwendung und Frequenz einzelner typografischer Mittel sowie dem Gestaltungsniveau des zielkulturellen Layouts zu achten.

Wird das Layout für das zielkulturelle bzw. zielsprachliche Publikat in der Ausgangskultur konzipiert und realisiert, ist meist die Translatorin die einzige Instanz, die verhindern kann, dass unreflektiert ausgangskulturelle Gestaltungskonventionen auf den Zieltext übertragen werden und dieser dann den Rezipienten buchstäblich „in fremdem Gewand“ begegnet.

Visuelle Kulturspezifik liegt vor, wenn sich Vorkommen, Ausgestaltung und Einsatzfrequenz einzelner typografischer Zeichen im Text oder typografische Relationen wie z.B. die visuelle Qualität eines Publikats in den beteiligten Translationskulturen unterscheiden. Prinzipiell kommen folgende Bereiche in Frage, auf die hier nur stichwortartig eingegangen werden kann (hierzu ausführlich Schopp 2005: 337-376).

Visuelle Kulturspezifik kann sich in den am Translationsprozess beteiligten nationalsprachlichen Schriftsystemen zeigen und ihren unterschiedlichen Transkriptionsprinzipien für z.B. russische oder arabische Namen oder im Charakter der in Ausgangstext und Zieltext präsenten Sprachsystemen und ihrer Auswirkung auf die Typografie der Texte (z. B. in Laufweite, Wortlänge und Satzart) und die Anordnung von Textelementen im Gesamtlayout (Beispiele bei Bokor 1998 sowie Schopp 2005: 342). Ein weiterer Punkt sind sprach- bzw. kulturspezifische typografische Zeichen (sprachspezifische Grafe) und die kulturspezifische Verwendung einzelner typografischer Zeichen in suprasegmentaler und tektonischer Funktion (z.B. zur Markierung direkter Rede), die einen wesentlichen Teil der Orthotypografie einer Schreibkultur bilden und sich u. U. auf die Les- und Sprechbarkeit des Textes auswirken. Von besonderer Bedeutung ist bei bestimmten Schriftarten der Schriftcharakter und seine kulturspezifisch assoziative Bewertung von peripheren grafischen Merkmalen (s.o. Abb. 3). Nicht vergessen werden soll die kulturspezifische Distribution von Allo- 
grafen wie dem langen und dem runden $S$ und den beiden unterschiedlich langen Gedankenstrichen (vgl. Schopp 2005: 356ff), denn auch diese gehören zum Bereich Orthotypografie, da sie u.U. den Lesefluss - wenn auch nur geringfügig - beeinträchtigen können.

Weniger erforscht ist derzeit noch der kulturspezifische Einsatz von Hervorhebungsmitteln sowie makrotypografischen Elementen (Layout-Elementen) wie den verschiedenen Satzarten (Block- und Flattersatz), dem symmetrischen und asymmetrischen Anordnungsprinzip, der Verwendung von Farben und Farbkombinationen, der Auswahl und Interpretation von Bildinhalten und dergleichen mehr. Schließlich spielen noch die Funktionalität eines in der Ausgangskultur konzipierten Layouts und seine qualitative Realisierung eine Rolle, die durch das bereits genannte „Überschreiben der layoutformatierten AT-Datei“ unberücksichtigt bleiben.

\section{Von der Anfrage bis »Gut-zum-Druck»}

Verstehen wir schriftliches translatorisches Handeln (Übersetzen) weniger als transoder interkulturelle Kommunikation per se, sondern eher als professionelles Handeln zur Anfertigung von Kommunikationsmitteln, die zu kommunikativen Zwecken in kommunikativen Aufgaben eingesetzt werden, bei denen Sprach- und Kulturgrenzen überwunden werden müssen, so ist es selbstverständlich, dass im Rahmen dieses Handelns alle Textebenen zu berücksichtigen sind, auch die visuelle - sei es, dass sie von einer Person (der Translatorin bzw. dem Translator) oder in Kooperation mit anderen Textfachleuten angefertigt werden. Aus diesem Grund spielt das Visuelle von Ausgangstext und Translat in allen Arbeitsphasen des professionellen Translationsprozesses eine nicht zu unterschätzende Rolle.

Wenn auch in der translatorischen Praxis durch die zunehmende Technik des „Überschreibens der layoutformatierten Ausgangstextdatei mit der Übersetzung“ der Eindruck erweckt wird, das Herstellungsverfahren eines Translats sei heute wesentlich einfacher als zu Zeiten traditioneller Druckverfahren, bleiben als translatorisches Aufgabenfeld prinzipiell die folgenden Arbeitsphasen, in denen die visuelle Gestalt des Translats eine mehr oder weniger wichtige Rolle spielt und mitberücksichtigt werden sollte.

1. Schon die aufgrund einer Anfrage notwendige Auftragsanalyse und Vorkalkulation muss die typografische Dimension des Textes berücksichtigen, da in Angebot und Vertrag der entsprechende Zeitaufwand als „Mehrwertdienstleistung“ (vgl. DIN EN 15038 2006: 5) zu berechnen ist. Dabei ist zu prüfen, ob die Wünsche des Auftraggebers mit der kommunikativen Funktion und den Rezeptionsbedingungen des zu erstellenden Translats im Einklang stehen und welche der drei Textebenen bearbeitet werden müssen (gegebenenfalls folgt daraus aufgrund des translatorischen Know-How-Vorsprungs die Pflicht zur Aufklärung und Beratung des Auftraggebers bzw. Bestellers). Bei Herstellung des zielkulturellen Publikats in der Ausgangskultur gehört hierzu die Fähigkeit, bereits jetzt einzuschätzen, ob das vorgesehene Layout in der Zielkultur „funktioniert“ oder ob Orthotypografie und andere typografische Parameter den Bedürfnissen der Zielsprache und -kultur angepasst werden müssen. Dann ist der Umfang der zu erbringenden Leistungen abzuschätzen und - abhängig vom Kompetenzprofil der Translatorin bzw. des Übersetzungsbüros - der u.U. notwendige Kooperationsbedarf festzustellen. Nach sorgfältiger Kalkulation aller 
Kosten sind im Angebot alle Leistungen aufzulisten und bei Bestellung vertraglich festzulegen.

2. Ausgangstextanalyse und Zieltextkonzeption: Hier geht es mit Hinblick auf die visuelle Gestalt von Ausgangstext und Zieltext und deren Integration in ein Publikat (1) um die Identifikation kulturspezifisch-bedeutungskonstituierender bzw. individueller, (autoren-) intendierter visueller Textelemente, (2) um die Überprüfung des ausgangskulturellen Layouts auf Funktionstauglichkeit in der Zielkultur und (3) um die Überprüfung und qualitative Bewertung eines in der Ausgangskultur konzipierten Layouts für ein zielkulturelles Publikat. Dabei ist es translatorische Aufgabe dafür zu sorgen, dass im Zieltext adäquate visuelle Lösungen geboten werden.

3. Translatorische Beratung in Layoutfragen (Konzeption des ZT-Layouts in der Ausgangskultur): Wie die Erfahrung zeigt, wird die visuelle Seite der Übersetzung nicht selten vernachlässigt, weil - wie im Falle von Mark Twains A Connecticut Yankee in Kings Arthur's Court - relevante visuelle Information des Ausgangstextes nicht in den Zieltext übernommen wird oder Gestaltungskonventionen der Zielkultur in Werbeagenturen und grafischen Betrieben der Ausgangskultur, in denen das Layout der Übersetzung hergestellt wird, nicht ausreichend bekannt sind. Oft kann nur die Translatorin als Text- und Kulturexpertin etwaige Probleme erkennen und den Auftraggeber darauf aufmerksam machen. Doch die von Holz-Mänttäri (1984: 115) geforderte „Mit-Verantwortung des Translators“ für das Layout erfordert eine spezielle Kompetenz, der entsprechende Inhalte in der Ausbildung zugrundeliegen müssen.

4. Visuelle Gestaltung des Translats: Die visuelle Gestaltung des Translats erfolgt von translatorischer Seite je nach Auftrag und Bestellung in unterschiedlichem Grad, wobei das Erstellen der Übersetzung mittels einer gängigen Textverarbeitungs-Software als selbstverständlich zu gelten hat und noch nicht als „Gestaltung“ im eigentlichen Sinne bezeichnet werden kann. Die endgültige Gestaltung des Translats reicht vom (1) Überschreiben der layoutformatierten AT-Datei über (2) der Reproduktion eines vorgegebenen Layouts durch Übernahme einer Layoutdatei und (3) der Adaption einer ausgangskulturellen Layoutvorlage bis zur (4) kreativen Gestaltung des ZT-Layouts, die in der Regel freilich eine gesonderte Ausbildung in Grafikdesign voraussetzt (vgl. Schopp 2005: 388ff).

5. Überprüfen der visuellen Textgestalt - Kundenkorrektur und "Imprimatur» bzw. »Gut zum Druck«: Diese letzte translatorische Arbeitsphase sollte bei Herstellung des zielkulturellen Publikats in der Ausgangskultur als selbstverständlicher Teil des gesamten Translationsprozesses betrachtet werden, d.h. dass die Translatorin an der Kundenkorrektur - bestehend in der Überprüfung von Orthografie und Orthotypografie - und der Erteilung des Imprimaturs beteiligt wird - ggf. in Kooperation mit dem Auftraggeber. Denn ihre Verantwortung für das Translat endet bei der Herstellung des Publikats in der Ausgangskultur nicht mit der Abgabe des Typoskripts bzw. der elektronischen Datei beim Auftraggeber, sondern erst, wenn sichergestellt ist, dass sich an der visuellen Gestalt des Zieltextes bzw. zielkulturellen Publikats nichts mehr ändert. Da aber der Auftraggeber bzw. Kunde in der Ausgangskultur in der Regel nicht selbst beurteilen kann, ob im Hinblick auf die zielkulturelle Funktion auf dem Korrekturabzug alles orthografisch und orthotypografisch korrekt ist, sind diese Aufgaben von translatorischer Seite zu übernehmen. 


\section{Wanted: die typografisch-translatorische Basiskompetenz}

Die Einbeziehung der visuellen Dimension des Translats in den Übersetzungsprozess setzt voraus, dass TranslatorInnen für typografische Fragen sensibilisiert sind und eine auf translatorische Bedürfnisse zugeschnittene typografische Basiskompetenz besitzen, die als Teil der professionellen translatorischen Grundkompetenz zu gelten hat (Schopp 2005). Diese sollte über das laientypografische Niveau hinausreichen, das heute dank Desktop-Publishing global verbreitet ist. Mehr noch: sie setzt voraus, dass das translatorische Berufsbild von der engen, inter- oder transkulturellen sprachlichen Handlung erweitert wird auf Zuständigkeit und Expertise für die holistische Anfertigung von Kommunikationsmitteln für inter- bzw. transkulturelle Kommunikationsaufgaben.

\section{ANMERKUNGEN}

1. Mein Vorschlag zu einem Oberbegriff für sowohl traditionell hergestellte Printmedien als auch auf elektronischem Weg publizierte digitale Medien.

2. So in der europäischen Übersetzungsnorm DIN EN 15038 (2006: 16).

3. Eine Ausnahme hiervon bilden sog. Random-Schriften wie die Beowulf, bei denen die Zeichengestalt nach einem Zufallsgenerator - allerdings in vorgegebenen Grenzen - variiert.

4. Dies und die aus dem Handlettering stammende Sitte, für die Textelemente vorwiegend Versalien (Großbuchstaben) zu verwenden, erschweren die Lesbarkeit nicht unerheblich.

5. Eine Übersicht des typografischen Instrumentariums findet sich bei Schopp (2005: 101-105).

6. Interessant ist, dass die sowohl das Original als auch die englische und finnische Übersetzung einen handschriftlich-gotischen Schriftcharakter verwenden, während die typografische deutsche Übersetzung an dieser Stelle in Frakturschrift gesetzt ist - andere Stellen der deutschen Übersetzung wie z.B. S. 42 weisen dann eine gotische Schrift auf. An sich ist die Wahl einer gebrochenen Schrift für die Goten ein Anachronismus, offensichtlich durch den Gleichklang der Wörter entstanden, hier jedoch dadurch motiviert, dass die Goten ganz offensichtlich deutsch-preußische Charakterzüge tragen.

7. Jegensdorf spricht von „linguistischer Kodierung“ (ibid.).

8. Dies erklärt auch die offensichtliche Vagheit in Holz-Mänttäris Textur-Begriff (s.u.).

9. Und wie hier Abb. 1 ein weiteres Mal zeigt.

10. Die stets mehr oder weniger stark vorhandene konnotative Wirkung der Grundschrift (vgl. (Jegensdorf 1980: 32f) soll hier unberücksichtigt bleiben.

11. Diese zweifache Kodierung entspricht allerdings nicht dem, was Jegensdorf (1980: 31f) darunter versteht.

12. „Systemoid“, weil Typografie nur systemähnlichen Charakter aufweist, da - anders als beim Zeichensystem Schrift - die einzelnen Mittel und Gestaltungsprinzipien stets polysem eingesetzt werden bzw. eine Funktion (z.B. die Markierung eines Abschnittes) durch unterschiedlichste Mittel visualisiert werden kann.

\section{LITERATUR}

BeInert, W. (2006): Typographie - Definition, Etymologie, Geschichte, <http//: www.typolexikon. de/t/typographie.html>, 12.9.2006.

BокоR, G. (1998): Translation and Typesetting, Translation Journal 1-2, <http//accurapid. com/journal/03type.htm>, 03.04.2002.

BRekle, H. E. (1997): Das typographische Prinzip. Versuch einer Begriffsklärung. In GutenbergJahrbuch 1997, 72, Jahrgang, Mainz, pp. 58-63.

DIN EN 15038 (2006): Übersetzungs-Dienstleistungen - Dienstleistungsanforderungen, Berlin, Beuth.

Емвасн, M. (2000): Skriptographie versus Typographie: Johannes Trithemius’ Schrift „De laude scriptorum", in Gutenberg-Jahrbuch 2000 75, Jahrgang, Mainz, pp. 132-144. 
Holz-Mänttäri, J. (1984): Translatorisches Handeln. Theorie und Methode, Helsinki, 1984. (= Annales Academiae Scientiarum Fennicae B 226).

Holz-MäntTärI, J. (1993): Textdesign - verantwortlich und gehirngerecht. In: Justa HolzMänttäri und Christiane Nord (Hrsg.). 1993. TRADUCERE NAVEM - Festschrift für Katharina Reiß zum 70. Geburtstag, Tampere (= studia translatologica ser. A vol. 3), pp. 301320.

KaINDL, K. (2004): Übersetzungswissenschaft im interdisziplinären Dialog, Am Beispiel der Comicübersetzung, Tübingen, (= Studien zur Translation, Bd.16), [Zugl.: Wien, Univ., Habil.-Schr., 1999]

Nöтн, W. (2000): Handbuch der Semiotik. 2., vollständig neu bearbeitete und erweiterte Auflage. Stuttgart, Weimar.

Oittinen, R. (1993): I Am Me - I Am Other: On the Dialogics of Translating for Children. Tampere (= Acta Universitatis Tamperensis ser. A vol. 386).

SAUSsuRE, F. de (1931/1967): Grundfragen der allgemeinen Sprachwissenschaft, Hrsg. von Charles Bally und Albert Sechehaye, Unter Mitwirkung von Albert Riedlinger übersetzt von Herman Lommel, 2. Aufl. mit neuem Register und einem Nachwort von Peter v. Polenz. Berlin.

Schopp, J. (1996): The Typographic Competence of the Translator - Visual Text Design and Desktop Publishing, in XIV World Congress of the Fédération Internationale des Traducteurs (FIT) February 1996, Melbourne, Australia, Proceedings Volume 1, pp. 189-195.

Schopp, J. F. (2002a.): Typographische Schrift als Mittel nationaler Identifikation. In: Valami más - Beiträge des Finnisch-Ungarischen Kultursemiotischen Symposiums „Zeichenhafte Aspekte der Veränderung" (25.-28.11.1998 Berlin, Franfurt/Oder, Slubice). Hrsg. von Eckhard Höfner, Hartmut Schröder u. Roland Wittmann. Frankfurt a.M. (= Nordeuropäische Beiträge aus den Human- und Gesellschaftswissenschaften, Bd. 22), pp. 95-126.

Schopp, J. F. (2002b): Typography and Layout as a Translation Problem. In: Translation: New Ideas for a New Century, Proceedings of the XVI FIT World Congress Vancouver, B.C., Canada 7 - 10 August, 2002, pp. 271-276.

Schopp, J. F. (2005): „Gut zum Druck“? - Typographie und Layout im Übersetzungsprozeß. Tampere (= Acta Universitatis Tamperensis 1117).

Tirttula, L. (1992): Puhuva kieli, Suullisen viestinnän erityispiirteitä, Helsinki.

Twyman, M. (1982): The graphic presentation of language. Information design journal 3/1, pp. 2-22.

Walker, S. (2001): Typography \& Language in Everyday Life: Prescriptions and Practices, Harlow.

WeHDE, S. (2000): Typographische Kultur - Eine zeichentheoretische und kulturgeschichtliche Studie zur Typographie und ihrer Entwicklung. Tübingen (= Studien und Texte zur Sozialgeschichte der Literatur, Bd.69).

Willberg, H. P. und F. Forssman (1997): Lesetypographie, Mainz.

\section{BILDNACHWEIS}

Abb. 1: Goscinny \& Uderzo: Astérix et les Goths. Neuilly/S/Seine: 1963, S. 33. - Asterix und die Goten. Stuttgart: 1990, S. 33. - Asterix and the Goths. London etc.: 1978, S. 33. - Asterix ja Gootit. Helsinki: 1977, S. 33.

Abb. 2: Schopp (2005: 61).

Abb. 3: Inserat zu den österreichischen Parlamentswahlen, veröffentlicht in Der Standard vom 9. Juni 1994.

Abb. 4: Titelseite des englischsprachigen und des deutschsprachigen Touristenführers von Kairo; vermutl. Ende 90er Jahre.

Abb. 5: Mark Twain: A Connecticut Yankee in King Arthur's Court. Berkley/Los Angeles/London: 1984 (The Mark Twain Library), Kap. 26. 